\title{
XLVI. On variations in the vertical due to elasticity of the earth's surface
}

\section{G.H. Darwin F.R.S.}

To cite this article: G.H. Darwin F.R.S. (1882) XLVI. On variations in the vertical due to elasticity of the earth's surface, Philosophical Magazine Series 5, 14:90, 409-427, DOI: $10.1080 / 14786448208628439$

To link to this article: http://dx.doi.org/10.1080/14786448208628439

曲 Published online: 28 Apr 2009.

Submit your article to this journal $[\pi$

Џ Article views: 3

Q View related articles $\square$ 


\title{
I.ONDON, EDINBURGH, AND DUBLIN \\ PHILOSOPHICAL MAGAZINE
}

\author{
AND \\ JOURNAL OF SCIENCE. \\ [FIFTH SERIES.] \\ $D E C E M B E R 1882$.
}

XLVI. On Variations in the Vertical due to Elasticity of the Earth's Surface. By G. H. DARwIN, F.R.S., formerly Fellow of Trinity College, Cambridge*.

1. On the Mechanical Effects of Barometric Pressure on the Earth's Surface.

THE remarks of Signore de Rossi, on the observed connexion between barometric storms and the disturbance of the vertical, have led me to make the following investigation of the mechanical effects which are caused by variations of pressure acting on an elastic surface. The results seem to show that the direct measurement of the lunar disturbance of gravity must for ever remain impossible.

The practical question is to estimate the amount of distortion to which the upper strata of the earth's mass are subjected, when a wave of barometric depression or elevation passes over the surface. The solution of the following problem should give us such an estimate.

Let an elastic solid be infinite in one direction, and be bounded in the other direction by an infinite plane. Let the surface of the plane be everywhere acted on by normal pressures and tractions, which are expressible as a simple harmonic function of distances measured in some fixed direction along

* Appendix to the Second Report of the Committee of the British Association on the Lunar Disturbance of Gravity. Read at the Meeting at Southampton, August 1882. Communicated by the Author.

Phil. Mag. S. 5. Vol. 14. No. 90. Dec. 1882. $2 \mathrm{E}$ 
the plane. It is required to find the form assumed by the surface, and generally the condition of internal strain.

This is clearly equivalent to the problem of finding the distortion of the earth's surface produced by parallel undulations of barometric elevation and depression. It is but a slight objection to the correctness of a rough estimate of the kind required, that barometric disturbances do not actually occur in parallel bands, but rather in circles. And when we consider the magnitude of actual terrestrial storms, it is obvious that the curvature of the earth's surface may be safely neglected.

This problem is mathematically identical with that of finding the state of stress produced in the earth by the weight of a series of parallel mountains. The solution of this problem has recently been published in a paper by me in the 'Philosophical Transactions' (part ii. 1882, pp. 187-230); and the solution there found may be adapted to the present case in a few lines.

The problem only involves two dimensions. If the origin be taken in the mean horizontal surface, which equally divides the mountains and valleys, and if the axis of $z$ be horizontal and perpendicular to the mountain-chains, and if the axis of $x$ be drawn vertically downwards, then the equation to the mountains and valleys is supposed to be

$$
x=-h \cos \frac{z}{b},
$$

so that the wave-length from crest to crest of the mountainranges is $2 \pi b$.

The solution may easily be found from the analysis of section 7 of the paper referred to. It is as follows:-

Let $\alpha, \gamma$ be the displacements at the point $x, z$ vertically downwards and horizontally ( $\alpha$ has here the opposite sign to the $\alpha$ of (44)). Let $w$ be the density of the rocks of which the mountains are composed, $g$ gravity, $v$ modulus of rigidity; then

where

$$
\left.\begin{array}{rl}
\alpha & =\frac{1}{2 v} b\left[x \frac{d \mathrm{~W}}{d x}-\mathrm{W}\right], \\
\gamma & =\frac{1}{2 v} b x \frac{d \mathrm{~W}}{d z}, \\
\mathrm{~W} & =-g w h e^{-x / b} \cos \frac{z}{b}
\end{array}\right\} . . . . .
$$

From these we have at once 


$$
\left.\begin{array}{rl}
\alpha & =\frac{g w h}{2 v} b\left(1+\frac{x}{b}\right) e^{-x / b} \cos \frac{z}{b}, \\
\gamma & =\frac{g w h}{2 v} x e^{-x / b} \sin _{\frac{z}{b}}^{z} \\
\frac{d \alpha}{d z} & =-\frac{g w h}{2 v}\left(1+\frac{x}{b}\right) e^{-x / b} \sin \frac{z}{b} .
\end{array}\right\} . . .
$$

The first of these gives the vertical displacement, the second the horizontal, and the third the inclination to the horizon of strata primitively plane.

At the surface,

$$
\left.\begin{array}{rl}
\alpha & =\frac{g w h}{2 v} b \cos \frac{z}{b}, \quad \gamma=0, \\
\frac{d \alpha}{d z} & =-\frac{g w h}{2 v} \sin \frac{z}{b} .
\end{array}\right\} . . . . .
$$

Hence the maximum vertical displacement of the surface is $\pm g w h b / 2 v$, and the maximum inclination of the surface to the horizon is

$$
\pm \operatorname{cosec} 1^{\prime \prime} \times g w h / 2 v \text { seconds of arc. }
$$

Before proceeding further, I shall prove a very remarkable relation between the slope of the surface of an elastic horizontal plane and the deflection of the plumb-line caused by the direct attraction of the weight producing that slope. This relation was pointed out to me by Sir William Thomson, when I told him of the investigation on which I was engaged; but I am alone responsible for the proof as here given. He writes that he finds that it is not confined simply to the case where the solid is incompressible; but in this paper it will only be proved for that case.

Let there be positive and negative matter distributed over the horizontal plane according to the law $w h \cos (z / b)$ : this

* It is easy to verify that these values of $\alpha$ and $\gamma$, together with the value $p=g w h e^{-x / b} \cos z / b$ for the hydrostatic pressure, satisfy all the conditions of the problem, by giving normal pressure gwh cos $z / b$ at the free surface of the infinite plane, and satisfying the equations of interual equilibrium throughout the solid. I take this opportunity of remarking that the paper from which this investigation is taken contains an error, inasmuch as the hydrostatic pressure is erroneously determined in section 1. The term - $W$ should be added to the pressure as determined in (3). This adds $W$ to the normal stresses $P, Q, R$ throughout the paper, but leaves the difference of stresses (which was the thing to be determined) unaffected. If the reader should compare the stresses as determined from the values of $\alpha, \gamma$ in the text above, and from the value of $p$ given in this note, with (38) of the paper referred to, he is warned to remember the missing term $\mathrm{W}$. 


\section{Mr. G. H. Darwin on Variations in the Vertical}

forms, in fact, harmonic mountains and valleys on the infinite plane. We require to find the potential and attraction of such a distribution of matter.

Now the potential of an infinite straight line, of line-density $\rho$, at a point distant $d$ from it, is well known to be $-2 \mu \rho \log d$, where $\mu$ is the attraction between unit masses at unit distance apart. Hence the potential V of the supposed distribution of matter at the point $x, z$ is given by

$$
\begin{gathered}
\mathrm{V}=-2 \mu w h \int_{-\infty}^{+\infty} \cos \frac{\zeta}{b} \log \sqrt{ }\left\{x^{2}+(\zeta-z)^{2}\right\} d \zeta \\
=-\mu w h b\left\{\left[\sin \frac{\zeta}{b} \log \left\{x^{2}+(\zeta-z)^{2}\right\}\right]_{-\infty}^{+\infty}-2 \int_{-\infty}^{+\infty} \frac{(\zeta-z) \sin (\zeta / b)}{x^{2}+(\zeta-z)^{2}} d \zeta\right\}
\end{gathered}
$$

It is not hard to show that the first term vanishes when taken between the limits.

Now put $t=\frac{\zeta-x}{x}$ so that $\sin \frac{\zeta}{b}=\sin \frac{t x}{b} \cos \frac{z}{b}+\cos \frac{t x}{b} \sin \frac{z}{b}$, and we have

$$
\mathrm{V}=2 \mu w h b \int_{-\infty}^{+\infty}\left(\sin \frac{t x}{b} \cos \frac{z}{b}+\cos \frac{t x}{b} \sin \frac{z}{b}\right) \frac{t d t}{1+t^{2}} .
$$

But it is known* that

$$
\int_{-\infty}^{+\infty} \frac{t \sin c t d t}{1+t^{2}}=\pi e^{-c}, \quad \int_{-\infty}^{+\infty} \frac{t \cos c t}{1+t^{2}} d t=0
$$

Therefore

$$
\mathrm{V}=2 \pi \mu w h b e^{-x / b} \cos \frac{z}{b} .
$$

If $g$ be gravity, $a$ earth's radius, and $\delta$ earth's mean density,

And

$$
2 \pi \mu=\frac{3 g}{2 a \delta} \text {. }
$$

$$
\mathrm{V}=\frac{3 g w h}{2 a \delta} b e^{-x / b} \cos \frac{z}{b} . \quad . \quad . \quad . \quad . \quad .
$$

The deflection of the plumb-line at any point on the surface denoted by $x=0$, and $z$, is clearly $d \mathrm{~V} / g d z$, when $x=0$. Therefore

$$
\text { the deflection }=-\frac{1}{g} \times \frac{3 g w h}{2 a \delta} \sin \frac{z}{b} . \quad .
$$

But from (2) the slope (or $\frac{d \alpha}{d z}$, when $z$ is zero) is

$$
-\frac{g u h}{2 v} \sin \frac{z}{b}
$$

See 'Todhunter's 'Integ. Calc.'; chapter on "I)efinite Integrals." 
Therefore deflection bears to slope the same ratio as $v / g$ to $\frac{1}{3} a \delta$. This ratio is independent of the wave-length $2 \pi b$ of the undulating surface, of the position of the origin, and of the azimuth in the plane of the line normal to the ridges and valleys. Therefore the proposition is true of any combination whatever of harmonic undulations; and as any inequality may be built up of harmonic undulations, it is generally true of inequalities of any shape whatever.

Now $a=6.37 \times 10^{8}$ centim., $\delta=5 \frac{2}{3}$; and $\frac{1}{3} a \delta=12.03 \times 10^{8}$ grammes per square centimetre. The rigidity of glass in gravitation-units ranges from $1.5 \times 10^{8}$ to $2.4 \times 10^{8}$. Therefore the slope of a very thick slab of the rigidity of glass, due to a weight placed on its surface, ranges from 8 to 5 times as much as the deflection of the plumb-line due to the attraction of that weight. Even with rigidity as great as steel (viz. about $8 \times 10^{8}$ ), the slope is $1 \frac{1}{2}$ times as great as the deflection.

A practical conclusion from this is that, in observations with an artificial horizon, the disturbance due to the weight of the observer's body is very far greater than that due to the attraction of his mass. This is in perfect accordance with the observations made by my brother and me with our pendulum in 1881, when we concluded that the warping of the soil by our weight when standing in the observing-room was a very serious disturbance, whilst we were unable to assert positively that the attraction of weights placed near the pendulum was perceptible. It also gives emphasis to the criticism we have made on M. Plantamour's observations-namely, that he does not appear to take special precautions against the disturbance due to the weight of the observer's body.

We must now consider the probable numerical values of the quantities involved in the barometric problem, and the mode of transition from the problem of the mountains to that of barometric inequalities.

The modulus of rigidity in gravitation-units (say grammes weight per square centimetre) is $v / g$. In the problem of the mountains, wh is the mass of a column of rock of one square centimetre in section and of length equal to the height of the crests of the mountains above the mean horizontal plane. In the barometric problem, wh must be taken as the mass of a column of mercury of a square centimetre in section and equal in height to a half of the maximum range of the barometer.

This maximum range is, I believe, nearly two inches, or, let us say, 5 centim.

The specific gravity of mercury is 13.6 ; and therefore $w h=34$ grammes.

The rigidity of glass is from 150 to 240 million grammes 


\section{Mr. G. H. Darwin on Variations in the Vertical}

per square centimetre, that of copper 540 , and of steel 843 millions.

I will take $v / g=3 \times 10^{8}$; so that the superficial layers of the earth are assumed to be more rigid than the most rigid glass. It will be easy to adjust the results afterwards to any other assumed rigidity.

With these data we have

$$
\frac{g w h}{2 v}=\frac{5 \cdot 67}{10^{8}}
$$

also

$$
\frac{648,000}{\pi} \times \frac{5 \cdot 67}{10^{8}}=0^{\prime \prime} \cdot 0117 \text {. }
$$

It seems not unreasonable to suppose that 1500 miles $\left(2 \cdot 4 \times 10^{8}\right.$ centim. $)$ is the distance from the place where the barometer is high (the centre of the anti-cyclone) to that where it is low (the centre of the cyclone). Accordingly the wave-length of the barometric undulation is $4.8 \times 10^{8}$ centim., and $b=4 \cdot 8 \times 10^{8} \div 6 \cdot 28$ centim., or, say, $b=\cdot 8 \times 10^{8}$ centim.

Thus, with these data,

$$
\frac{g w h}{2 v} b=4 \cdot 5 \text { centim. }
$$

We thus see that the ground is 9 centim. higher under the barometric depression than under the elevation.

If the sea had time to attain its equilibrium slope, it would stand $5 \times 13 \cdot 6$, or 68 centim. lower under the high pressure than under the low. But as the land is itself depressed 9 centim., the sea would apparently only be depressed 59 centim. under the high barometer.

It is probable that, in reality, the larger barometric inequalities do not linger quite long enough over particular areas to permit the sea to attain everywhere its due slope, and therefore the full difference of water-level can only be attained occasionally.

On the other hand, the elastic compression of the ground must take place without any sensible delay. Thus it seems probable that the elastic compression of the ground must exercise a very sensible effect in modifying the apparent depression or elevation of the sea under high and low barometer.

It does not appear absolutely chimerical that at some future time, when both tidal and barometric observations have attained to great accuracy, an estimate might thus be made of the average modulus of rigidity of the upper 500 miles of the earth's mass.

Even in the present condition of barometric and tidal infor- 
mation, it might be interesting to make a comparison between the computed height of tide and the observed height, in connexion with the distribution of barometric pressure. It is probable that India would be the best field for such an attempt, because the knowledge of Indian tides is more complete than that for any other part of the world. On the other hand, we shall see in the following section that tidal observations on coast-lines of continents are liable to disturbance, so that an oceanic island would be a more favourable site.

It has already been shown that the maximum apparent deflection of the plumb-line, consequent on the elastic compression of the earth, amounts to $0^{\prime \prime} \cdot 0117$; and this is augmented to $0^{\prime \prime} \cdot 0146$, when we include the true deflection due to the attraction of the air. It is worthy of remark that this result is independent of the wave-length of the barometric inequality, and thus we get rid of one of the conjectural data.

Thus, if we consider the two cases of high pressure to right and low to left, and of low pressure to right and high to left, we see that there will be a difference in the position of the plumb-line relatively to the earth's surface of $0^{\prime \prime} \cdot 0292$. Even if the rigidity of the upper strata of the earth were as great as that of steel, there would still be a change of $0^{\prime \prime} \cdot 011$.

A deflection of magnitude such as $0^{\prime \prime} \cdot 03$ or $0^{\prime \prime} \cdot 01$ would have been easily observable with our instrument of last year; for we concluded that a change of $\frac{1}{200}$ of a second could be detected when the change occurred rapidly.

It was stated in our previous Report that at Cambridge the calculated amplitude of oscillation of the plumb-line due directly to lunar disturbance of gravity amounts to $0^{\prime \prime} 0216$. Now, as this is less than the amplitude due jointly to elastic compression and attraction, with the assumed rigidity (300 millions) of the earth's strata, and only twice the result if the rigidity be as great as that of steel, it follows almost certainly that from this cause alone the measurement of the lunar disturbance of gravity mnst be impossible with any instrument on the earth's surface.

Moreover the removal of the instrument to the bottom of the deepest known mine would scarcely sensibly affect the result, because the flexure of the strata at a depth so small, conipared with the wave-length of barometric inequalities, is scarcely different from the flexure of the surface.

The diurnal and periodic oscillations of the vertical observed by us were many times as great as those which have just been computed; and therefore it must not be supposed that more than a fraction, say perhaps a tenth, of those oscillations was due to elastic compression of the earth. 
The Italian observers could scarcely with their instruments detect deflections amounting to $\frac{1}{10}$ of a second; so that the observed connexion between barometric oscillation and seismic disturbance must be of a different kind.

It is not surprising that in a volcanic region the equalization of pressure, between imprisoned fluids and the external atmosphere, should lead to earthquakes.

If there is any place on the earth's surface free from seismic forces, it might be possible (if the effect of tides as computed in the following section could be eliminated) with some such instrument as ours, placed in a deep mine, to detect the existence of barometric disturbance many hundreds of miles away. It would of course for this purpose be necessary to note the positions of the sun and moon at the times of observation, and to allow for their attraction.

\section{On the Disturbance of the Vertical near the Coasts of Continents due to the Rise and Fall of the Tide.}

Consider the following problem:-

On an infinite horizontal plane, which bounds in one direction an infinite incompressible elastic solid, let there be drawn a series of parallel straight lines, distance $l$ apart. Let one of these be the axis of $y$, let the axis of $z$ be drawn in the plane, perpendicular to the parallel lines, and let the axis of $x$ be drawn vertically downwards through the solid.

At every point of the surface of the solid, from $z=0$ to $l$, let a normal pressure gwh $(1-2 z / l)$ be applied; and from $z=0$ to $-l$ let the surface be free from forces. Let the same distribution of force be repeated over all the pairs of strips into which the surface is divided by the system of parallel straight lines. It is required to determine the strains caused by these forces.

Taking the average over the whole surface, there is neither pressure nor traction, since the total traction on the half-strips subject to traction is equal to the total pressure on the halfstrips subject to pressure.

The following is the analogy of this system with that which we wish to discuss : the strips subject to no pressure are the continents, the alternate ones are the oceans, $g$ is gravity, $w$ the density of water, and $h$ the height of tide above mean water on the coast-line.

We require to find the slope of the surface at every point, and the vertical displacement.

It is now necessary to bring this problem within the range of the results used in the last section. In the first place, it is convenient to consider the pressures and tractions as caused by 
mountains and valleys whose outline is given by $x=-h(1-2 z / l)$ from $z=0$ to $l$, and $x=0$ from $z=0$ to $-l$. To utilize the analysis of the last section, it is necessary that the mountains and valleys should present a simple-harmonic outline. Hence the discontinuous function must be expanded by Fourier's method. Known results of that method render it unnecessary to have recourse to the theorem itself. It is known that

$$
\begin{aligned}
\pm \frac{1}{2} \pi-\frac{1}{2} \theta & =\sin \theta+\frac{1}{2} \sin 2 \theta+\frac{1}{3} \sin 3 \theta+\ldots \\
-\frac{1}{2} \theta & =-\sin \theta+\frac{1}{2} \sin 2 \theta-\frac{1}{3} \sin 3 \theta+\ldots \\
\frac{1}{2} \pi \mp \theta & =\frac{4}{\pi}\left\{\cos \theta+\frac{1}{3^{2}} \cos 3 \theta+\frac{1}{5^{2}} \cos 5 \theta+\ldots\right\},
\end{aligned}
$$

the upper sign being taken for values of $\theta$ between the infinitely small positive and $+\pi$, and the lower for values between the infinitely small negative and $-\pi$.

Adding these three series together, we have

$2\left\{\frac{1}{2} \sin 2 \theta+\frac{1}{4} \sin 4 \theta+..\right\}+\frac{4}{\pi}\left\{\cos \theta+\frac{1}{3^{2}} \cos 3 \theta+\frac{1}{5^{2}} \cos 5 \theta+..\right\}$ equal to $\pi-2 \theta$ from $\theta=0$ to $+\pi$, and equal to zero from $\theta=0$ to $-\pi$. Hence the required expansion of the discontinuous function is

$$
\left.\begin{array}{l}
-\frac{2 h}{\pi}\left\{\frac{1}{2} \sin 2 \theta+\frac{1}{4} \sin 4 \theta+\ldots\right\}, \\
-\frac{4 h}{\pi^{2}}\left\{\cos \theta+\frac{1}{3^{2}} \cos 3 \theta+\frac{1}{5^{2}} \cos 5 \theta+\ldots\right\},
\end{array}\right\} .
$$

where

$$
\theta=\frac{\pi z}{l} ; . . \quad . \quad . \quad . \quad .
$$

for it vanishes from $z=-l$ to 0 , and is equal to $-h(1-2 z / l)$ from $z=0$ to $+l$.

Now, looking back to the analysis of the preceding section, we see that, if the equation to the mountains and valleys had been $x=-h \sin (z / b)$, $\alpha$ would have had the same form as in (2), but of course with sine for cosine, and $\gamma$ would have changed its sign and a cosine would have stood for the sine. Applying then the solution (2) to each term of our expansion separately, and only writing down the solution for the surface at which $x=0$, we have at once that $\gamma=0$, and

$$
\begin{aligned}
\alpha & =\frac{g w h}{\pi v} \frac{l}{\pi}\left\{\frac{1}{2^{2}} \sin 2 \theta+\frac{1}{4^{2}} \sin 4 \theta+\frac{1}{6^{2}} \sin 6 \theta+\ldots\right\} \\
& +\frac{g w h}{\pi v} \cdot \frac{2 l}{\pi^{2}}\left\{\cos \theta+\frac{1}{3^{3}} \cos 3 \theta+\frac{1}{5^{3}} \cos 5 \theta+\ldots\right\} .
\end{aligned}
$$


The slope of the surface is $\frac{d \alpha}{d z}$ or $\frac{\pi}{l} \frac{d \alpha_{0}}{d \theta^{3}}$; thus

$$
\left.\begin{array}{rl}
\frac{d \alpha}{d z} & =\frac{g w h}{\pi v}\left\{\frac{1}{2} \cos 2 \theta+\frac{1}{4} \cos 4 \theta+\frac{1}{6} \cos 6 \theta+\ldots\right\} \\
& -\frac{g w h}{\pi v} \cdot \frac{2}{\pi}\left\{\sin \theta+\frac{1}{3^{2}} \sin 3 \theta+\frac{1}{5^{2}} \sin 5 \theta+\ldots\right\}
\end{array}\right\}
$$

The formulæ (8) and (9) are the required expressions for the vertical depression of the surface and for the slope.

It is interesting to determine the form of surface denoted by these equations. Lat us suppose, then, that the units are so chosen that $g w h l / \pi^{2} v$ may be equal to one. Then (8) and (9) become

$$
\begin{aligned}
\alpha & =\frac{1}{2^{2}} \sin 2 \theta+\frac{1}{4^{2}} \sin 4 \theta+\ldots+\frac{2}{\pi}\left\{\frac{1}{1^{3}} \cos \theta+\frac{1}{3^{3}} \cos 3 \theta+\ldots\right\} \\
\frac{d \alpha}{d \theta} & =\frac{1}{2} \cos 2 \theta+\frac{1}{4} \cos 4 \theta+\ldots-\frac{2}{\pi}\left\{\frac{1}{1^{2}} \sin \theta+\frac{1}{3^{2}} \sin 3 \theta+\ldots\right\} .
\end{aligned}
$$

When $\theta$ is zero or $\pm \pi, d \alpha / d \theta$ becomes infinite, which denotes that the tangent to the warped horizontal surface is vertical at these points. The verticality of these tangents will have no place in reality, because actual shores shelve, and there is not a vertical wall of water when the tide rises, as is supposed to be the case in the ideal problem. We shall, however, see that in practical numerical application, the strip of sea-shore along which the solution shows a slope of more than $1^{\prime \prime}$ is only a small fraction of a millimetre. Thus this departure from reality is of no importance whatever.

When $\theta=0$ or $\pm \pi$,

$$
\alpha=\frac{2}{\pi}\left\{\frac{1}{1^{3}}+\frac{1}{3^{3}}+\frac{1}{5^{3}}+\ldots\right\}=\frac{2}{\pi} \times 1 \cdot 052=\cdot 670,
$$

being + when $\theta=0$, and - when $\theta= \pm \pi$.

When $\theta= \pm \frac{1}{2} \pi, \alpha$ vanishes; and therefore midway in the ocean and on the land there are nodal lines, which always remain in the undisturbed surface, when the tide rises and falls. At these nodal lines, defined by $\theta= \pm \frac{1}{2} \pi$,

$$
\begin{aligned}
\frac{d \alpha}{d \theta} & =-\frac{1}{2} \log _{e} 2 \mp \frac{2}{\pi}\left\{\frac{1}{1^{3}}-\frac{1}{3^{3}}+\frac{1}{5^{3}}-\ldots\right\} \\
& =-\cdot 3466 \mp \cdot 6168=-.9634 \text { and }+\cdot 2702 .
\end{aligned}
$$

Thus the slope is greater at mid-ocean than at mid-land. By assuming $\theta$ successively as $\frac{1}{6} \pi, \frac{1}{4} \pi, \frac{1}{3} \pi$, and summing arithmetically the strange series which arise, we can, on pay- 
ing attention to the manner in which the signs of the series occur, obtain the values of $\alpha$ corresponding to $0, \pm \frac{1}{6} \pi, \pm \frac{1}{4} \pi$, $\pm \frac{2}{6} \pi, \pm \frac{3}{6} \pi, \pm \frac{4}{6} \pi, \pm \frac{5}{6} \pi, \pm \frac{6}{6} \pi$. The resulting values, together with the slopes as obtained above, are amply suffiient for drawing a figure, as shown annexed.

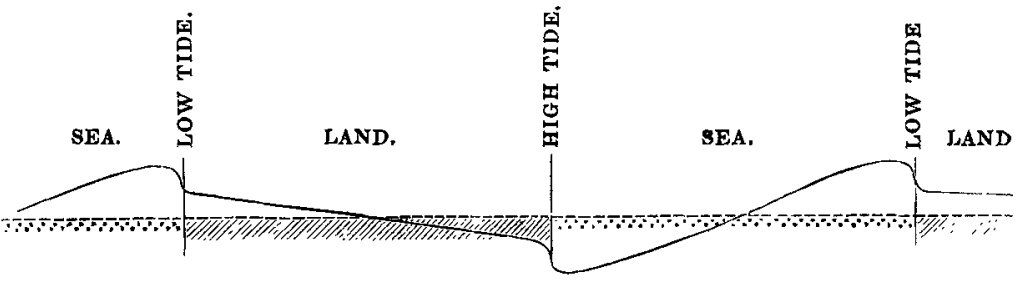

The straight line is a section of the undisturbed level, the shaded part being land, and the dotted sea. The curve shows the distortion, when warped by high and low tide as indicated.

The scale of the figure is a quarter of an inch to $\frac{1}{6} \pi$ for the abscissas, and a quarter of an inch to unity for the ordinates; it is of course an enormous exaggeration of the flexure actually possibly due to tides.

It is interesting to note that the land-regions remain very nearly flat, rotating about the nodal line, but with slight curvature near the coasts. It is this curvature, scarcely perceptible in the figure, which is of most interest for practical application.

The series (8) and (9) are not convenient for practical calculation in the neighbourhood of the coast, and they must be reduced to other forms. It is easy, by writing the cosines in their exponential form, to show that

$\cos \theta+\frac{1}{2} \cos 2 \theta+\frac{1}{3} \cos 3 \theta+\ldots=-\log _{e}\left( \pm 2 \sin \frac{1}{2} \theta\right)$, .

$\cos \theta-\frac{1}{2} \cos 2 \theta+\frac{1}{3} \cos 3 \theta-\ldots=\log _{e}\left(2 \cos \frac{1}{2} \theta\right)$, . .

where the upper sign in (13) is to be taken for positive values of $\theta$ and the lower for negative.

For the small values of $\theta$ with which alone we are at present concerned, the series (13) becomes $-\log _{e}( \pm \theta)$ and the lower $\log _{e} 2$.

Taking half the difference and half the sum of the two series, we have

$\frac{1}{2} \cos 2 \theta+\frac{1}{4} \cos 4 \theta+\ldots \ldots=-\frac{1}{2} \log ( \pm \theta)-\frac{1}{2} \log 2,$.

$\cos \theta+\frac{1}{3} \cos 3 \theta+\frac{1}{5} \cos 5 \theta+=-\frac{1}{2} \log ( \pm \theta)+\frac{1}{2} \log 2$. .

Integrating (16) with regard to $\theta$, and observing that the 
constant introduced on integration is zero, we have $\sin \theta+\frac{1}{3^{2}} \sin 3 \theta+\frac{1}{5^{2}} \sin 5 \theta+\ldots=-\frac{1}{2} \theta[\log ( \pm \theta)-1]+\frac{1}{2} \theta \log 2$. Then, from (15) and (17),

$$
\begin{gathered}
\frac{1}{4} \cos 2 \theta+\frac{1}{4} \cos 4 \theta+\ldots-\frac{2}{\pi}\left\{\sin \theta+\frac{1}{3^{2}} \sin 3 \theta+\ldots\right\} \\
=-\frac{1}{2}\left(1-\frac{2 \theta}{\pi}\right) \log ( \pm \theta)-\frac{1}{2}\left(1+\frac{2 \theta}{\pi}\right) \log 2-\frac{\theta}{\pi} .
\end{gathered}
$$

Integrating (15), and observing that the constant is zero, we have

$$
\frac{1}{2^{2}} \sin 2 \theta+\frac{1}{4^{2}} \sin 4 \theta+\ldots=-\frac{1}{2} \theta[\log ( \pm \theta)-1]-\frac{1}{2} \theta \log 2 .
$$

Integrating (17), and putting in the proper constant to make the left side vanish when $\theta=0$, we have

$$
\begin{array}{r}
\frac{1}{1^{3}}+\frac{1}{3^{3}}+\frac{1}{5^{3}}+\ldots-\left(\frac{1}{1^{3}} \cos \theta+\frac{1}{3^{3}} \cos 3 \theta+\ldots\right) \\
=-\frac{1}{4} \theta^{2} \log ( \pm \theta)+\frac{1}{4} \theta^{2}\left(\frac{3}{2}+\log 2\right) . .
\end{array}
$$

For purposes of practical calculation, $\theta$ may be taken as so small that the right-hand side of (18) reduces to $-\frac{1}{2} \log ( \pm 2 \theta)$, and the right-hand sides of (19) and (20) to zero.

Hence, by (8) and (9), we have in the neighbourhood of the coast,

$$
\left.\begin{array}{rl}
\alpha & =\frac{g w h}{\pi v} \times \frac{2 l}{\pi^{2}}\left[\frac{1}{1^{3}}+\frac{1}{3^{3}}+\frac{1}{5^{3}}+\ldots\right], \\
& =\frac{g w h}{\pi v} \times \frac{l}{\pi^{2}} \times 2 \cdot 1037, \\
\frac{d \alpha}{d z} & =-\frac{g w h}{2 \pi v} \log _{e} 10 \log _{10} \frac{2 \pi z}{l} .
\end{array}\right\}
$$

I shall now proceed to compute from the formulæ (21) the depression of the surface and the slope, corresponding to such numerical data as seem most appropriate to the terrestrial oceans and continents.

Considering that the tides are undoubtedly augmented by kinetic action, we shall be within the mark in taking $h$ as the semi-range of equilibrium tide. At the equator the lunar tide has a range of about 53 centim., and the solar tide is very nearly half as much. Therefore at spring-tides we may take $h=40$ centim. It must be noticed that the highness of the tides (say 15 or 20 feet) near the coast is due to the shallowing of the water, and it would not be just to take such values 
as representing the tides over large areas; $w$, the density of the water, is of course unity.

If we suppose it is the Atlantic Ocean and the shores of Europe with Africa, and of North and South America, which are under consideration, it is not unreasonable to take $l$ as 3900 miles, or $6.28 \times 10^{8}$ centim. Then $2 \pi z / l=z \times 10^{-8}$.

Taking $v / g$ as $3 \times 10^{8}$ (that is to say, assuming a rigidity greater than that of glass), we have for the slope in seconds of are, at a distance $z$ from the sea-shore,

$$
\begin{array}{r}
\operatorname{cosec} 1^{\prime \prime} \times \frac{40}{2 \pi \times 3 \times 10^{8}} \times \log _{e} 10 \times\left(8-\log _{10} z\right) \\
=0^{\prime \prime} .01008\left(8-\log _{10} z\right) . \quad . \quad .
\end{array}
$$

From this the following table may be computed by simple multiplication:-

$$
\begin{aligned}
& \text { Distance from } \\
& \text { mean water-mark. Slope. } \\
& 1 \text { centim. }=1 \text { centim. . . } 00^{\prime / 0} 0806 \\
& 10, \quad=10 \% . . . \quad 0706 \\
& 10^{2} \% \quad=1 \text { metre. . . . } .0605 \\
& 10^{3} \% \quad=10 \text { metres. . . . } 0504 \\
& 10^{4} ", \quad=100, \ldots . . . \quad . \quad 0403 \\
& 10^{5} \% \quad=1 \text { kilom. . . . } \quad 0302 \\
& 10^{6}, \quad=10, \ldots . . . .0202 \\
& 2 \times 10^{6} \text { centim. }=20 \% \text {. . . . } 0170 \\
& 5 \times 10^{6}, \quad=50 \% \text {. . . . } 0131 \\
& 10^{7} \text { centim." }=100, " . . . \quad .0101
\end{aligned}
$$

On considering the formula (22), it appears that $z$ must be a very small fraction of a millimetre before the slope becomes even as great as $1^{\prime}$. 'This proves that the rounded nick in the surface, which arises from the discontinuity of pressure at our ideal mean water-mark, is excessively small ; and the vertical displacement of the surface is sensibly the same, when measured in centimetres, on each side of the nick, in accordance with the first of (21).

The result (5) of section 1 shows that, with rigidity $3 \times 10^{8}$, the true deflection of plumb-line due to attraction of the water is a quarter of the slope. Hence an observer in a gravitational observatory at distance $z$ from mean water-mark, would note deflections from the mean position of the vertical $1 \frac{1}{4}$ times as great as those computed above; and as high water changes to low, there would be oscillations of the vertical $2 \frac{1}{2}$ times as great. We thus get the practical results in the following table:- 


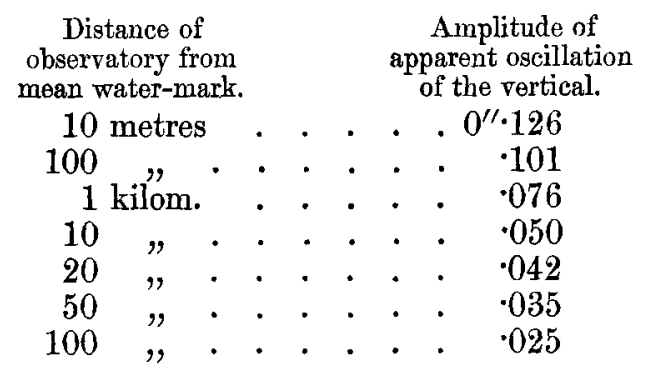

It follows, from the calculations made for tracing the curve, that halfway across the continent (that is to say, 3142 kilometres from either coast) the slope is

$$
\frac{648,000}{\pi} \times \frac{g w h}{\pi v} \times \cdot 2703 \text { second of are }=0^{\prime \prime} \cdot 00237,
$$

and the range of apparent oscillation is $0^{\prime \prime} \cdot 006$.

In these calculations the width of the sea is taken as 6283 kilometres. If the sea be narrower, then, to obtain the same deflections of the plumb-line, the observatory must be moved nearer the sea in the same proportion as the sea is narrowed. If, for example, the sea were 3142 kilometres wide, then at 10 kilometres from the coast the apparent amplitude of deflection would be $0^{\prime \prime} \cdot 042$. If the range of tide is greater than that here assumed (viz. 80 centim.), the results must be augmented in the same proportion. And, lastly, if the rigidity of the rock be greater or less than the assumed value (viz. $3 \times 10^{8}$ ), the part of the apparent deflection depending on slope must be diminished or increased in the inverse proportion to the change in rigidity.

I think there can be little doubt that in narrow seas the tides are generally much greater than those here assumed; and it is probable that at a gravitational observatory actually on the sea-shore on the sonth coast of England, apart from seismic changes, perceptible oscillations of the vertical would be noted.

Sir William Thomson has made an entirely independent estimate of the probable deflection of the plumb-line at a seaside gravitational observatory*. He estimates the attraction of a slab of water 10 feet thick (the range of tide), 50 miles broad perpendicular to the coast, and 100 miles long parallel with coast, on a plummet 100 yards from the low-water mark, and opposite the middle of the 100 miles of length. He thinks this estimate would very roughly represent the state of things

* Thomson and Tait's 'Natural Philosophy,' 818. 
say at St. Alban's Head. He finds, then, that the deflection of the plumb-line as high tide changes to low would be 4000000 of the unit angle, or $0^{\prime \prime} \cdot 050$. The general theorem proved above, as to the proportionality of slope to attraction, shows that, with rigidity $3 \times 10^{8}$ for the rocks of which the earth is formed, the apparent deflection of the plumb-line would amount to $0 / / 25$.

It is just possible that a way may in this manner be opened for determining the modulus of rigidity of the upper 100 or 200 miles of the earth's surface, although the process would be excessively laborious. The tides of the British Channel are pretty well known; and therefore it would be possible by very laborious quadratures to determine the deflection of the plumb-line due to the attraction of the tide at any time at a chosen station. If, then, the deflection of the plumb-line could be observed at that station (with corrections applied for the positions of the sun and moon), the ratio of the calculated to the observed and corrected deflection, together with the known value of the earth's radius and mean density, form the materials for computing the rigidity. But such a scheme would be probably rendered abortive by just such comparatively large and capricious oscillations of the vertical as we, M. d'Abbadie, and others have observed.

It is interesting to draw attention to some observations of M. d'Abbadie on the deflections of the vertical due to tides. His observatory (of which an account was given in the Report for 1881) is near Hendaye, in the Pyrenees, and stands 72 metres above and 400 metres distant from the sea. $\mathrm{He}$ writes*:-

“'J'ai réuni 359 comparaisons d'observations spéciales faites lors du maximum du flot et du jusant; 243 seulement sont favorables à la théorie de l'attraction exercée par la masse des eaux, et l'ensemble des résultats pour une différence moyenne do marées égale à 2.9 mètres donne un résultat moyen de $0^{\prime \prime} \cdot 56$ ou $0^{\prime \prime} \cdot 18$ pour le double de l'attraction angulaire vers le Nord-Ouest. Ceci est conforme à la théorie, car lez différences observées doivent être partagées par moitié, selon la loi de la réflexion; mais comme il y a toujours de l'inattendu dans les expériences nouvelles, on doit ajouter que sur les 116 comparaisons restantes il y en a eu 57 où le flot semble repousser le mercure au lieu de l'attirer. Mes résultats ont été confirmés pendant l'hiver dernier par M. l'abbé Artus, qui a eu la patience de comparer ainsi 71 flots et 73 jusants consécutifs, de janvier à mars 1880. Lui aussi a trouvé un tiers environ * "Recherches sur la Verticale," Ann. de la Soc. Scient. de Bruxelles, 1881. 


\section{Mr. G. H. Darwin on Variations in the Vertical}

de cas défavorables à nos théories admises. On est donc en droit d'affirmer que si la mer haute attire le plus souvent le pied du fil à plomb, il y a une, et peut-être plusieurs, autres forces en jeu pour faire varier sa position."

We must now consider the vertical displacement of the land near the coast. In (21) it is shown to be

$$
\alpha_{0}=\frac{g w h}{\pi v} \times \frac{l}{\pi^{2}} \times 2 \cdot 1037
$$

where $\alpha_{0}$ indicates the displacement corresponding to $z=0$.

With the assumed values, $h=40, v=3 \times 10^{8}, l=6.28 \times 10^{8}$, I find $\alpha_{0}=5.684$ centim. Hence the amplitude of vertical displacement is 11.37 centim. As long as $h l$ remains constant this vertical displacement remains the same; hence the high tides of 10 or 15 feet which are actually observed on the coasts of narrow seas must probably produce vertical oscillations of quite the same order as that computed.

If the land falls, the tide of course rises higher on the coastline than it would do otherwise; hence the apparent height of tide would be $h+\alpha_{0}$. But this show there is more water resting on the earth than according to the estimated value $h$; hence the depression of the soil is greater in the proportion $1+\alpha_{0} / h$ to unity; this again causes more tide, which reacts and causes more depression, and so on. Thus on the whole the augmentation of tide due to elastic yielding is in the ratio of

$$
1+\frac{\alpha_{0}}{h}+\left(\frac{a_{0}}{h}\right)^{2}+\left(\frac{\alpha_{0}}{h}\right)^{3}+\ldots \text { or } \frac{1}{1-\alpha_{0} / h} \text { to unity. }
$$

This investigation is conducted on the equilibrium theory ; and it neglects the curvature of the sea-bed, assuming that there is a uniform slope from mid-ocean to the sea-coast. The figure shows that this is not rigorously the case; but it is quite near enough for a rough approximation. The phenomena of the short-period tides are so essentially kinetic that the value of this augmentation must remain quite uncertain; but for the long-period tides (the fortnightly and monthly elliptic) the augmentation must correspond approximately with the ratio

$$
1:\left(1-\frac{g w l}{\pi^{3} v} \times 2 \cdot 1037\right)
$$

The augmentation in narrow seas will be small; but in the Atlantic Ocean the augmenting factor must agree pretty well with that which I now compute*.

* Sir William Thomson has pointed out to me, since the meeting of the Association, that this angmentation will only hold true in the cases of certain distributions of land. 
With the previous numerical values we have $\alpha_{0} / h$ (which is independent of $h$ ) equal to $\cdot 1421$, and $1-\alpha_{0} / h=\cdot 8579=\frac{6}{7}$ very nearly.

Thus the long-period tides may probably undergo an augmentation at the coasts of the Atlantic in some such ratio as 6 to 7.

The influence of this kind of elastic yielding is antagonistic to that reduction of apparent tide which must result from an elastic vielding of the earth's mass as a whole.

The reader will probably find it difficult to estimate what degree of probability of correctness there is in the conjectural value of the rigidity, which has been used in making the numerical calculations in this paper. The rigidity has not been experimentally determined for many substances; but a great number of experiments have been made to find Young's modulus. Now, in the stretching of a bar or wire the compressibility plays a much less important part than the rigidity; and the formula for Young's modulus shows that for an incompressible elastic solid the modulus is equal to three times the rigidity*. Hence a third of Young's modulus will form a good standard of comparison with the assumed rigidity, namely $3 \times 10^{8}$ grammes weight per square centimetre. The following are a few values of a third of Young's modulus and of rigidity, taken from the tables in Sir William Thomson's article on Elasticityt in the Encyclopedia Britannica:-

Material. $\quad \begin{aligned} & \text { A third of Young's modulus and } \\ & \text { rigidity in terms of } 10^{\circ} \text { grammes } \\ & \text { weight per square centimetre. }\end{aligned}$
Stone . . . . . About $1 \cdot 2$
Slate . . . . About 3 to 4.
Glass . . . . Rigidity $1 \cdot 5$ to $2 \cdot 4$.
Ice . . . . . $4 \cdot 7$.
Copper. . . . 4 , and rigidity $4 \cdot 6$ to $5 \cdot 4$.
Steel . . . . 7 to 10 , and rigidity $8 \cdot 4$.

It will be observed that the assumed rigidity 3 is probably a pretty high estimate in comparison with that of the materials of which we know the superficial strata to be formed.

It is shown, in another paper read before the Association at this meeting, that the rigidity of the earth as a whole is probably as great as that of steel. That result is not at all inconsistent with the probability of the assumption that the upper strata have only a rigidity a little greater than that of glass.

* Thomson and Tait's 'Natural Philosophy, $\$ 683$.

+ Also published separately by Black (Edinburgh).

Phil. Mag. S. 5. Vol. 14. No. 90. Dec. 1882. 


\section{On Gravitational Observatories.}

In the preceding sections estimates have been made of the amount of distortion which the upper strata of the earth probably undergo from the shifting weights corresponding to barometric and tidal oscillations. These results appear to me to have an important bearing on the utility of gravitational observatories.

It is not probable, at least for many years to come, that the state of tidal and barometric pressure, for a radius of 500 miles round any spot on the earth's surface, will be known with sufficient accuracy to make even a rough approximation to the slope of the surface a possibility. And were these data known, the heterogeneity of geological strata would form a serious obstacle to the possibility of carrying out such a computation. It would do little in relieving us from these difficulties to place the observatory at the bottom of a mine.

Accordingly the prospect of determining experimentally the lunar disturbance of gravity appears exceedingly remote; and I am compelled reluctantly to conclude that continuous observations with gravitational instruments of very great delicacy are not likely to lead to results of any great interest. It appears likely that such an instrument, even in the most favourable site, would record incessant variations of which no satisfactory account could be given. Although I do not regard it as probable that such a delicate instrument should be adopted for regular continuous observations, yet, by choosing a site where the flexure of the earth's surface is likely to be great, it is conceivable that a rough estimate might be made of the average modulus of elasticity of the upper strata of the earth for one or two hundred miles from the surface.

These conclusions, which I express with much diffidence, are by no means adverse to the utility of a coarser gravitational instrument, capable, let us say, of recording variations of level amounting to $1^{\prime \prime}$ or $2^{\prime \prime}$. If barometric pressure, tidal pressure, and the direct action of the sun and moon combine together to make apparent slope in one direction, then, at an observatory remote from the sea-shore, that slope might perhaps amount to a quarter of a second of arc. Such a disturbance of level would not be important compared with the minimum deviations which could be recorded by the supposed instrument.

It would then be of much value to obtain continuous systematic observations, after the manner of the Itglians, of the seismic and slower quasi-seismic variations of level. 
I venture to predict that at some future time practical astronomers will no longer be content to eliminate variations of level merely by taking means of results, but will regard corrections derived from a special instrument as necessary to each astronomical observation.

XLVII. New Views of Mr. George H. Darwin's Theory of the Evolution of the Earth-Moon System, considered as to its bearing on the question of the Duration of Geological Time. By ihe Rev. Samuer Haughton, M.D., Fellow of Trinity College, Dublin*.

TT has been tacitly assumed, even so far back as the times 1 of Newton and Clairaut, that the earth and planets have passed through a liquid condition (owing to former great heat) before assuming the solid condition which some, at least, of them now possess.

Laplace, in his nebular hypothesis, also assumes the former existence of this liquid condition; and it is openly asserted by all geologists who believe that the earth consists of a solid crust (more or less thick), reposing upon a fluid or viscous nucleus.

It has been proved by Sir William Thomson, following out the views of the late Mr. Hopkins, that the present condition of the earth, taken as a whole, is such that it must be regarded as being more rigid than glass or steel, possibly more rigid than any terrestrial substance under the surface-conditions of pressure.

The following considerations show that it may be fairly doubted whether the earth or any other planet ever existed in a fluid condition.

1. The possibility of the equilibrium of the rings of Saturn, on the supposition that they are either solid or liquid, has been more than doubted, and the most probable hypothesis respecting them is, that they consist of swarms of discrete meteoric stones.

2. It is difficult to understand the low specific gravity of Jupiter and the other outer planets, on the supposition that they are either solid or liquid; for we know of no substance light enough to form them $\dagger$. If the outer planets consist of

* From the 'American Journal of Science ' for November 1882. Read before the Mathematical Section of the American Association for the Advancement of Science, at Montreal, August 1882.

$\uparrow$ The force of this argument could not be felt before the revelations of the spectroscope, because at that time there was no proof that the whole universe was composed of the same simple substances, and those very limited in number. 